\title{
ARTICLE
}

\section{IL-17-dependent SIgA-mediated protection against nasal Bordetella pertussis infection by live attenuated BPZE1 vaccine}

Luis Solans ${ }^{1,2,3,4}$, Anne-Sophie Debrie ${ }^{1,2,3,4}$, Lisa Borkner ${ }^{5}$, Nacho Aguiló ${ }^{6,7}$, Anaïs Thiriard ${ }^{1,2,3,4}$, Loic Coutte ${ }^{1,2,3,4}$, Santi Uranga ${ }^{6,7}$, François Trottein ${ }^{1,2,3,4}$, Carlos Martín ${ }^{6,7}$, Kingston H. G. Mills ${ }^{5}$ and Camille Locht ${ }^{1,2,3,4}$

BPZE1 is a live attenuated Bordetella pertussis vaccine for nasal administration to mimic the natural route of infection. Here, we studied the mechanism of BPZE1-induced immunity in the murine nasal cavity in contrast to acellular vaccine (aPV), although both vaccines protected against lung colonization. Transfer of splenocytes or serum from BPZE1-vaccinated or aPV-vaccinated mice protected naïve mice against lung colonization but not against nasal colonization. However, transfer of nasal washes from BPZE1vaccinated mice resulted in protection against nasal colonization, which was lost in IgA-deficient or poly-lg receptor-deficient mice, indicating that it depends on secretory $\lg \mathrm{A}(\mathrm{S} \lg \mathrm{A})$ induction induced in the nose. BPZE1-induced protection against nasal colonization was long-lived despite the relatively rapid decay of SIgA, indicating a potent BPZE1-induced local memory response, likely due to $\mathrm{CD}^{+}$tissue-resident memory T cells induced in the nose by BPZE1. These cells produced interleukin-17 (IL-17), known to be important for SIgA secretion. Furthermore, BPZE1 failed to protect $1 / 17^{-1-}$ mice against nasal colonization by $B$. pertussis and induced only background levels of nasal SIgA. Thus, our results show important differences in the protective mechanism between the upper and the lower murine respiratory tract and demonstrate an IL-17-dependent SIgA-mediated mechanism of BPZE1induced protection against $B$. pertussis nasopharyngeal colonization.

Mucosal Immunology (2018) 11:1753-1762; https://doi.org/10.1038/s41385-018-0073-9

\section{INTRODUCTION}

Pertussis or whooping cough is a severe and highly contagious respiratory disease, mainly caused by Bordetella pertussis. ${ }^{1}$ Although it is most severe and can be life threatening in very young children, it also affects other age groups, and can cause significant morbidity in adolescents and adults, including the elderly. ${ }^{2}$ Despite a current global vaccination coverage of $>85 \%$, ${ }^{3}$ the disease has not been controlled in any country and has even made a spectacular comeback in areas where vaccine coverage has reached close to $100 \%,{ }^{4}$ illustrating the shortcomings of current vaccination programs.

Many reasons for this failure to control pertussis have been proposed, including pathogen adaptation to escape vaccineinduced immunity, fast waning of immunity, especially since the switch from whole-cell to acellular vaccines (aPV), and the failure of pertussis vaccines, in particular aPV, to prevent infection and spread of B. pertussis. ${ }^{5}$ Several investigations have now shown that apparently symptom-less $B$. pertussis infections are much more frequent than previously appreciated, even in fully vaccinated populations, ${ }^{6-10}$ and recent modeling studies have suggested that asymptomatic transmission may be the major driver of the resurgence of pertussis. ${ }^{11}$ In contrast to current vaccines, prior infection is able to prevent $B$. pertussis infection and transmission, as shown in baboon studies. ${ }^{12}$
We have developed a live attenuated $B$. pertussis vaccine to be delivered nasally. This vaccine, named BPZE1, was constructed by genetically removing or inactivating three toxins: tracheal cytotoxin, dermonecrotic toxin, and pertussis toxin (PTX). ${ }^{13}$ It was found to be safe in pre-clinical models and highly effective in protecting mice against $B$. pertussis after a single nasal administration (for a review see Locht and Mielcarek et al.. ${ }^{14}$ ) It is now in clinical development and was shown to be safe in humans, able to transiently colonize the human respiratory tract and to induce immune responses in all colonized individuals. ${ }^{15}$

It has recently been tested in baboons, where it induced strong protection against pertussis disease after a single vaccination and reduced nasopharyngeal B. pertussis colonization by $99.998 \%$ over non-vaccinated baboons, ${ }^{16}$ a performance that by far exceeded that of three administrations of a human dose of aPV and wholecell vaccine. ${ }^{12}$ However, the mechanism responsible for the protection against nasal colonization has not been addressed yet. In this study, we used the mouse model to examine the immune mechanism of BPZE1-mediated protection against nasal infection by $B$. pertussis, which allowed us to identify $B$. pertussisspecific secretory $\lg \mathrm{A}(\mathrm{S} \lg \mathrm{A})$ in the nasal cavity as the main effector molecule for nasal protection.

\footnotetext{
${ }^{1}$ Center of Infection and Immunity of Lille, Institut Pasteur de Lille, 59019 Lille, France; ${ }^{2}$ Inserm U1019, 59019 Lille, France; ${ }^{3}$ CNRS UMR8204, 59019 Lille, France; ${ }^{4}$ Univ. Lille, 59019 Lille, France; ${ }^{5}$ Immune Regulation Research Group, School of Biochemistry and Immunology, Trinity College Dublin, Trinity Biomedical Science Institute, Dublin 2, Ireland; ${ }^{6}$ Grupo de Genética de Micobacterias, Dpto. de Microbiología, Medicina Preventiva y Salud Pública, C/Domingo Miral s/n, 50009 Zaragoza, Spain and ${ }^{7}$ CIBER Enfermedades Respiratorias, Instituto de Salud Carlos III, 28029 Madrid, Spain

Correspondence: Luis Solans (solansbernad@gmail.com)
}

Received: 20 November 2017 Revised: 5 July 2018 Accepted: 23 July 2018

Published online: 16 August 2018 
a Lungs protection in BalB/c mice

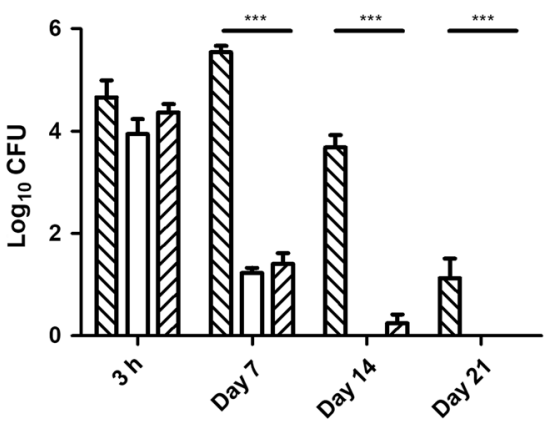

C

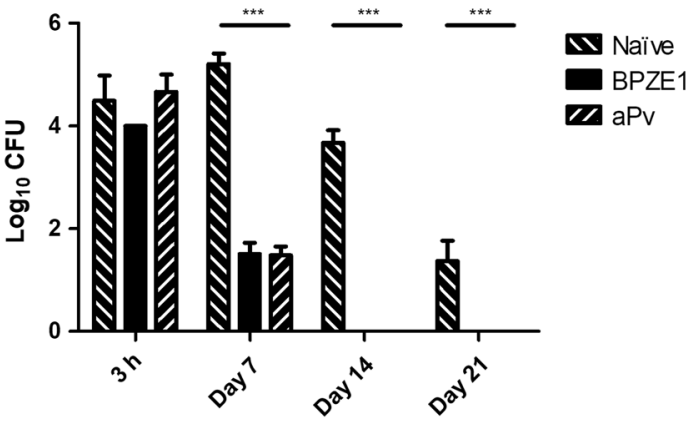

b

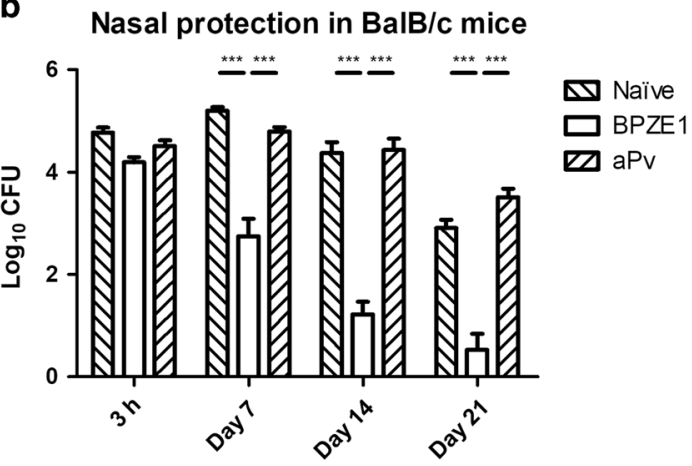

d Nasal protection in C57BL/6 mice

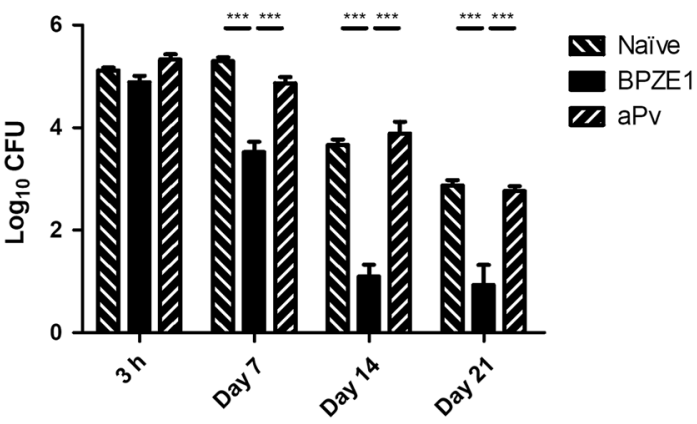

Fig. 1 Protection induced by BPZE1 or aPV against B. pertussis colonization of Balb/c and C57BL/6 mouse lungs and noses. Groups of Balb/c or C57BL/6 mice were vaccinated once with BPZE1 or twice with aPV at a 4-week interval. Eight weeks after the first vaccination, mice were challenged with B. pertussis BPBCTA1 and CFU levels were measured at the indicated time points in the lungs (a, c) and noses (b, d) of Balb/c $(\mathbf{a}, \mathbf{b})$ and $\mathrm{C} 57 \mathrm{BL} / 6$ (c, d) mice. $N=3$ for the $3 \mathrm{~h}$ time point and $N=5$ for the other time points. Bars represent mean values and graph represents data combined from three independent experiments. ${ }^{* * *} p<0.001$ by two-way ANOVA and Bonferroni post hoc test

\section{RESULTS}

BPZE1, but not aPV, induces protection against nasal infection by B. pertussis

In most published studies using mice to evaluate the potency of pertussis vaccines, the animals were infected intranasally or by aerosol, and the bacterial burden in the lungs was determined at different time points. This model, in which mice receive two administrations of $1 / 5$ of a human aPV dose, is routinely used as it correlates with aPV efficacy in human clinical trials. ${ }^{17,18}$ When Balb/c (Fig. 1a, b) and C57BL/6 (Fig. 1c, d) mice were vaccinated with a single nasal dose of $10^{6}$ colony-forming unit (CFU) BPZE1, a dose established according to previous dose-responses studies, ${ }^{19}$ or two administrations of $1 / 5$ of a human aPV dose at a 4-week interval and challenged with virulent $B$. pertussis BPBCTA1 8 weeks after the first vaccination, strong protection with either vaccine was seen in the lungs (Fig. 1a, c), as expected from previous studies. ${ }^{13}$

However, when the bacterial load in the noses were examined, BPZE1 was found to induce strong protection against nasal colonization by virulent $B$. pertussis, whereas aPV failed to control the infection in the nasal cavity (Fig. 1b, d). Three hours after challenge infection, colony counts in the noses were similar to those detected in the lungs, which is different from other studies. These differences may be due to the fact that here the mice were infected by nasal drops in contrast to aerosol infection in other studies. Nasal drops may more efficiently lead to nasal colonization. Furthermore, we have examined total bacterial burden in nasal homogenates, compared to other studies in which nasal washes (NWs) were examined. When we compared NW to nasal homogenates, we indeed saw a roughly 10-fold lower CFU recovery in the NW (data not shown).
Transfer of immune serum or splenocytes from vaccinated mice provides protection in the lungs but not in the nose

The analysis of Balb/c serum antibody responses to whole $B$. pertussis extracts revealed that nasal administration of BPZE1 induced both serum immunoglobulin $\mathrm{G}(\mathrm{IgG})$ and immunoglobu$\operatorname{lin} A(\lg A)$, whereas subcutaneous administration of aPV only induced serum IgG (Fig. 2a, b). The IgG isotypes induced by the two vaccines were also different. Whereas aPV primarily induced serum anti-B. pertussis IgG1 (Fig. 2C), indicative of a T-helper 2 (Th2)-type immune response, the lgG isotype of the BPZE1vaccinated mice was primarily lgG2a (Fig. 2d), lgG2b (Fig. 2e), and lgG3 (Fig. 2f), indicative of Th1-type and Th17-type immune responses. This is consistent with our previous results showing that aPV and BPZE1 preferentially induce Th2-producing and Th1 cytokine-producing $T$ cells upon stimulation with $B$. pertussis antigens, respectively. ${ }^{13}$ Interestingly, we found here that aPV preferentially induced IL-17 responses to PTX and filamentous hemagglutinin (FHA), whereas BPZE1 preferentially induced IL-17 to pertactin, especially in the lungs, but also, to a minor degree, in the spleen (Supplementary Figure 1). Similar lgG isotype profiles were seen in C57BL/6 mice (Supplementary Figure 2). When antibodies were measured against the three antigens present in aPV, FHA, PTX, and pertactin, IgG were measurable against all three antigens both in BPZE1-vaccinated and aPV-vaccinated mice, whereas IgA could only be detected against pertactin in BPZE1-vaccinated mice, both in Balb/c (Supplementary Figure 3) and in C57BL/6 mice (Supplementary Figure 4).

In order to assess the protective effect of these antibodies against nasal infection by $B$. pertussis, serum from aPV-vaccinated or BPZE1-vaccinated mice was injected intraperitoneally into severe combined immunodeficiency (SCID) mice, which were intranasally challenged with virulent BPSM $24 \mathrm{~h}$ later. Whereas the transfer of either serum resulted in significant protection in the 
a

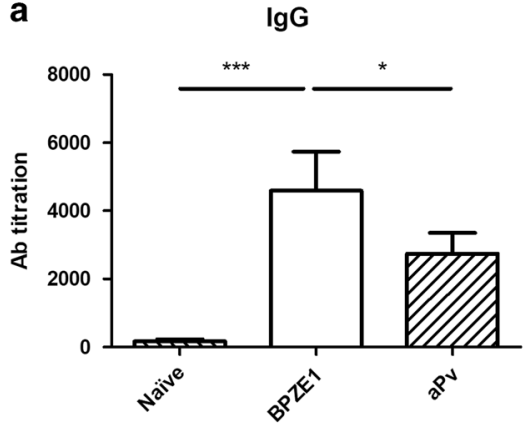

d

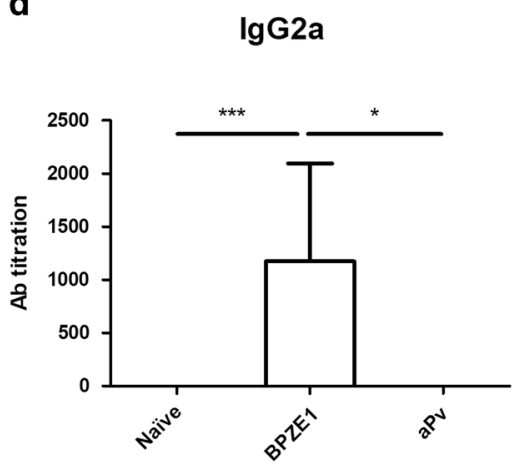

b

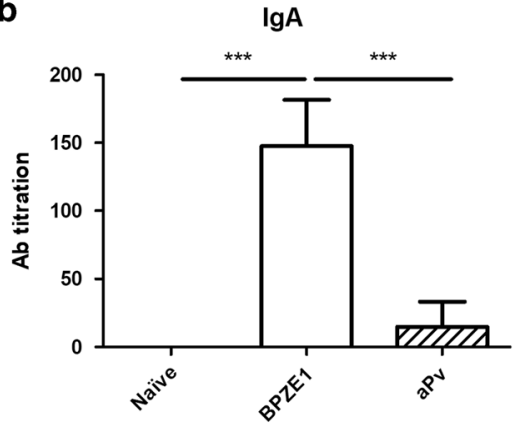

e

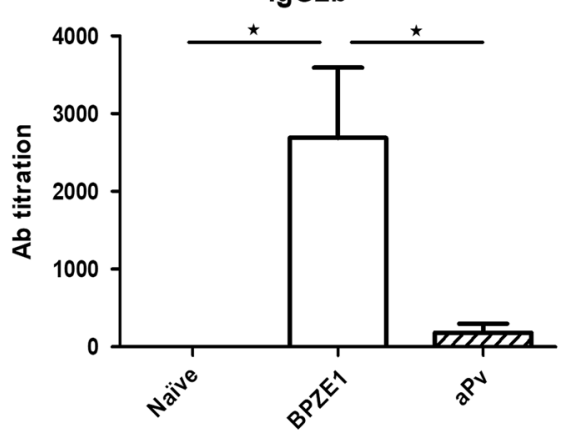

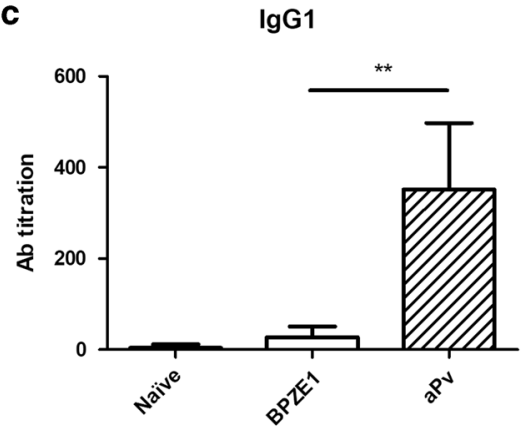

$\mathbf{f}$

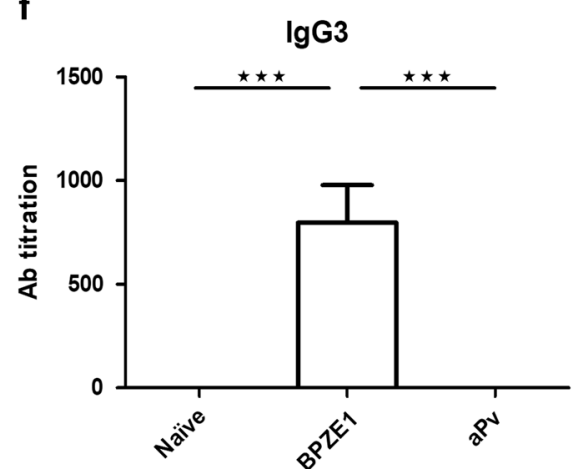

Fig. 2 Serum antibody induction by BPZE1 or aPV vaccination in Balb/c mice. Groups of Balb/c mice were vaccinated once with BPZE1, twice with aPV at a 4 weeks interval, or left unvaccinated (Naïve) as indicated. Eight weeks after the first vaccination, mice were sacrificed and serum was collected. Total $\operatorname{lgG}(\mathbf{a}), \lg \mathrm{A}(\mathbf{b}), \lg \mathrm{l} 1$ (c), $\lg \mathrm{g} 2 \mathrm{a}(\mathbf{d}), \operatorname{lgG} 2 \mathrm{~b}(\mathbf{e})$, and $\lg \mathrm{G} 3(\mathbf{f})$ titers were measured against BPSM lysate protein extract. $N=5$. Bars represent mean values and graph represents data combined from three independent experiments. ${ }^{*} p<0.05 ;{ }^{* *} p<0.01 ;{ }^{* * *} p<0.001$ by one-way ANOVA and Bonferroni post hoc test

lungs, compared to transfer of serum from naïve mice (Fig. 3a), none of them conferred protection in the nose (Fig. 3b).

Since protection against $B$. pertussis may also be cellmediated, ${ }^{20}$ we also examined the protective effect of splenocyte transfer. Similar to the antisera, splenocytes from mice vaccinated with either BPZE1 or aPV transferred significant levels of protection to SCID mice in the lung, whereas splenocytes from naïve mice did not (Fig. 3c). In contrast, none of them transferred protection in the nasal cavity (Fig. 3d), although a trend of a decreased bacterial burden was seen when splenocytes from BPZE1-vaccinated mice were transferred. However, this did not reach statistical significance.

Transfer of slgA in NWs from BPZE1-vaccinated mice provides protection against nasal $B$. pertussis infection

Since neither serum antibodies nor spleen cells from BPZE1vaccinated mice could transfer protection against nasal infection by $B$. pertussis, we reasoned that nasal protection may require the induction of local antibodies by the vaccine. We first measured anti- $B$. pertussis antibodies in the nasal secretions of BPZE1vaccinated mice. As shown in Fig. 4a, BPZE1 vaccination induced barely detectable anti-B. pertussis IgG levels in NWs, whereas significant amounts of anti- $B$. pertussis $\lg A$ titers were induced (Fig. 4b). These IgA were genuine SIgA, as they were not detected in NWs of polymeric immunoglobulin receptor-deficient $\left(\mathrm{Pigr}^{-1-}\right)$ mice (Fig. 4a, b).

NWs from BPZE1-vaccinated mice were then instilled into the noses of naïve mice, which were subsequently challenged with virulent $B$. pertussis. Seven days after infection, the bacterial load in the noses of these recipient mice was significantly lower than in the noses of mice that had received NW from naïve mice (Fig. 4c). No protection was seen when NW from BPZE1-vaccinated Pigr $^{-1-}$ mice were transferred to naïve mice, indicating that the protection depended on antibodies actively transported via plgR.
BPZE1-induced protection against nasal B. pertussis infection depends on SIgA

As significant BPZE1-induced protection against nasal infection by $B$. pertussis could be transferred by anti-B. pertussis IgA-containing NWs, we further investigated the role of plgR on BPZE1-mediated protection in the nose. C57BL/6 and Pigr $^{-1-}$ mice were intranasally vaccinated with BPZE1 and challenged 2 months later with BPBCTA1. Three hours after challenge, vaccinated and nonvaccinated mice contained the same bacterial burden in the nose (Fig. 5). A significant decrease in bacterial load was observed in the vaccinated C57BL/6 mice 7 and 21 days after infection, compared to the non-vaccinated mice, whereas this decrease was much less pronounced in the Pigr $^{-1-}$ mice, indicating that the active transport of immunoglobulins into the mucosal lumen is important for nasal protection.

Since in addition to $\lg \mathrm{A}$ plgR can also transport other immunoglobulin isotypes, it was important to confirm the role of IgA in nasal protection. We therefore vaccinated $\lg A^{-1-}$ mice with BPZE1 and measured the bacterial burden after challenge with virulent $B$. pertussis BPBCTA1. Seven and twenty-one days after infection with BPBCTA1, the $\lg A^{-1-}$ mice were significantly less protected than the C57BL/6 mice (Fig. 5). Together, these results demonstrate that BPZE1-induced protection against nasal infection by $B$. pertussis is mediated by SIgA secreted into the nasal cavity. There was no difference in BPZE1-mediated protection between $\lg \mathrm{A}^{-/-}$and $\mathrm{Pigr}^{-/-}$mice at day 7 after infection, and a slight but significant difference was seen at day 21 after infection (slightly better protection in the $\mathrm{IgA}^{-/-}$than in the $\mathrm{Pigr}^{-/-}$mice). It is thus likely that isotypes other than IgA may contribute to protection against nasal infection by $B$. pertussis, but this would best be at a lesser extent. 
a

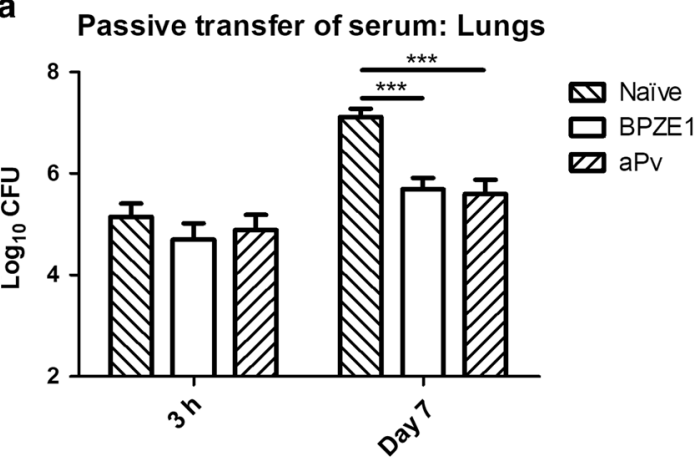

C

Passive transfer of splenocytes: Lungs

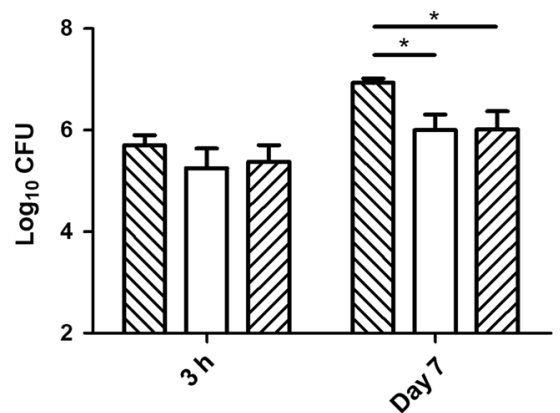

b

Passive transfer of serum: Nose

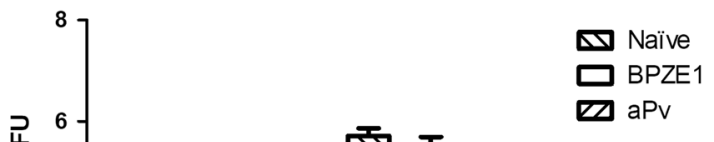

d

Passive transfer of splenocytes: Nose

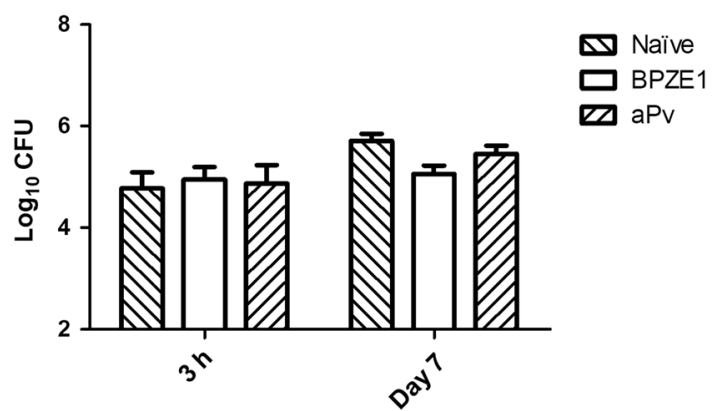

Fig. 3 Protection by passive transfer of serum and splenocytes from BPZE1-vaccinated or aPV-vaccinated Balb/c mice in SCID mice. Balb/c mice were vaccinated once with BPZE1 and twice with aPV at a 4-week interval (naïve) as indicated. Eight weeks after the first vaccination, mice were sacrificed and serum and splenocytes were collected and transferred intraperitoneally into SCID mice. Twenty-four hours later SCID mice were challenged with $B$. pertussis BPSM and CFU counts were measured in the lungs $(\mathbf{a}, \mathbf{c})$ and the noses (b, d) of the mice which had received the serum $(\mathbf{a}, \mathbf{b})$ or the splenocytes $(\mathbf{c}, \mathbf{d}) . N=3$ for the $3 \mathrm{~h}$ time point and $N=5$ for the day 7 time point. Bars represent mean values and graph represents data combined from three independent experiments. ${ }^{*} p<0.05$ and ${ }^{* * *} p<0.001$ by two-way ANOVA and Bonferroni post hoc test

Long-term persistence of BPZE1-induced protection in the nasal cavity

In order to study the persistence of BPZE1-induced protection in the nasal cavity, Balb/c and C57BL/6 mice were vaccinated with a single dose of BPZE1 and challenged with virulent $B$. pertussis 10 months after vaccination. Significant protection against nasal colonization by $B$. pertussis was still observed for both mouse strains 10 months after vaccination (Fig. 6a, c), although long-term protection appeared to be stronger in Balb/c mice than in C57BL/6 mice. However, when NWs from Balb/c or C57BL/6 mice vaccinated 10 months earlier were transferred intranasally into naïve mice prior to challenge, no difference in bacterial burden was observed 7 days after challenge between mice that had received NWs from vaccinated mice and those that had received NWs from naïve mice (Fig. 6b, d). Furthermore, B. pertussis-specific IgA had dropped to background levels 10 months after BPZE1 vaccination (Supplementary Figure $5 \mathrm{~A}$ ). Together, these observations are consistent with the notion that $\lg A$ are relatively shortlived, ${ }^{21}$ but that, in contrast, the memory of mucosal immunity is long-lived. ${ }^{22}$

BPZE1 induces resident memory $T$ cells in the nose

Virulent $B$. pertussis infection has been shown to induce resident memory $T$ (Trm) cells in the lungs of mice. ${ }^{23}$ As these cells are long lived and can rapidly expand upon secondary antigen encounter, they may be the prime candidates to sustain mucosal immune memory. We therefore examined the ability of BPZE1 to induce Trm cells in the nose. The numbers of Trm cells, as defined by the $\mathrm{CD} 4{ }^{+} \mathrm{CD} 69^{+} \mathrm{CD}_{103}{ }^{+}$phenotype were increased 14 days after BPZE1 administration in both Balb/c and C57BL/6 mice (Fig. 7a, b).
Furthermore, the Trm cells produced IL-17 and, to a lesser extent, IFN- $\gamma$ (Fig. 7c, d).

BPZE1-induced nasal protection depends on IL-17

Since BPZE1 induces IL-17-producing Trm in the noses of mice, we next determined the role of IL-17 in BPZE1-mediated nasal protection. We thus vaccinated $1 / 17^{-1-}$ mice with BPZE1 and measured the bacterial load in the noses $3 \mathrm{~h}$ and 7 days after challenge with virulent $B$. pertussis BPBCTA1. All mice, vaccinated or not, contained comparable amounts of BPBCTA1 bacteria in the nose $3 \mathrm{~h}$ after infection. At day 7 after infection, the vaccinated $\| 17^{-1-}$ mice carried significantly more BPBCTA1 organisms in their noses than the vaccinated C57BL/6 mice (Fig. 8). Anti-B. pertussis IgA levels in the NWs of BPZE1-vaccinated $1117^{-/-}$mice were substantially reduced (Supplementary Figure 5B). However, protection was not completely abolished by the lack of IL-17, since compared to the non-vaccinated animals, vaccinated $1117^{-1-}$ mice still contained approximately two orders of magnitude less CFU in their noses at day 7 after infection, as was also seen in the $\mathrm{Pigr}^{-1-}$ and in the $\lg \mathrm{A}^{-1-}$ mice (Fig. 8).

\section{DISCUSSION}

Pertussis is essentially an upper respiratory disease, and the bacteria can be found in large numbers mostly between the cilia on the surface of the epithelial cells lining the trachea, ${ }^{1}$ although in severe cases patients may suffer from bronchopneumonia, which may be due to secondary infections or to $B$. pertussis itself. ${ }^{24}$ Bordetella pertussis initiates its infection by attachment to the epithelial cells of the upper airway, thereby qualifying it as a typical mucosal pathogen. Therefore, it would be expected that 
a

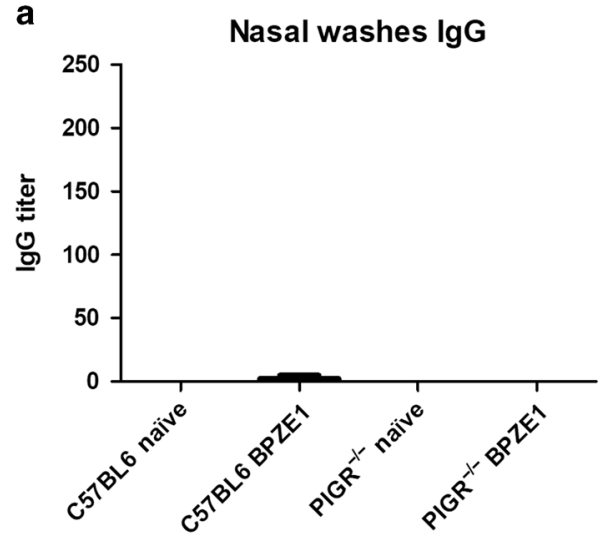

b

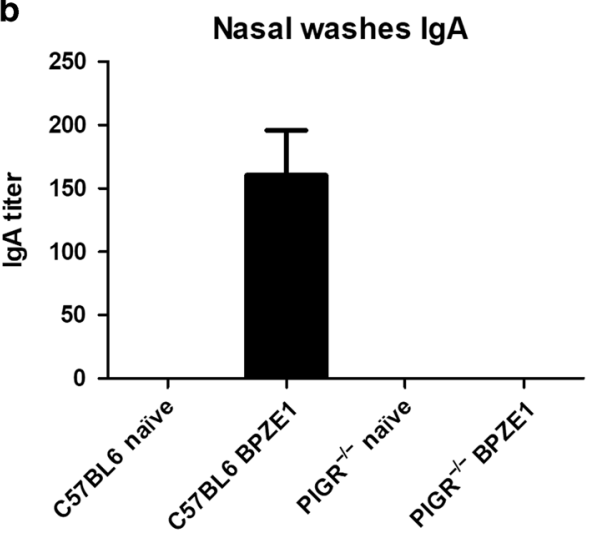

C

Challenge of C57BL6 after nasal wash transfer

Nose

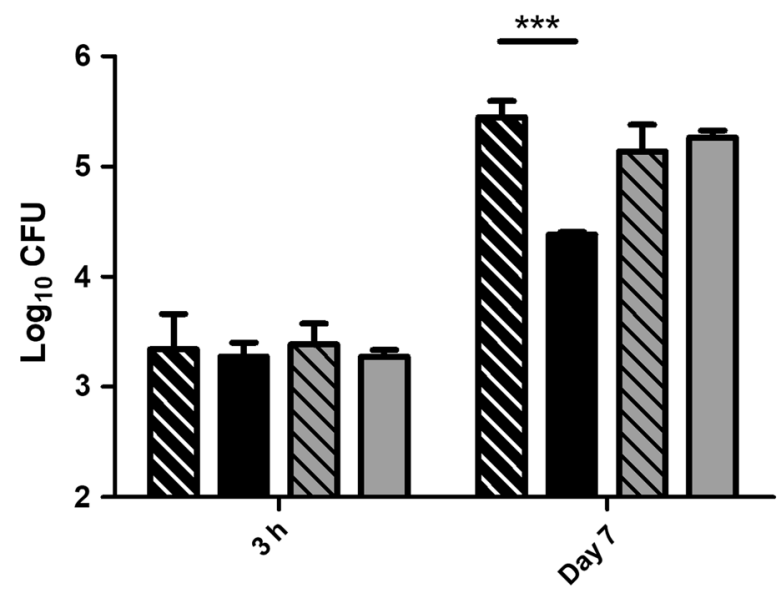

I) NW transfer from C57BL6 naïve

NW transfer from C57BL6 BPZE1

$\triangle$ NW transfer from $\mathrm{PIGR}^{-/-}$naïve

$\square$ NW transfer from PIGR ${ }^{-/-}$BPZE1

Fig. 4 Antibody titers in nasal washes and protection by passive transfer of nasal washes against nasal colonization by $B$. pertussis. Groups of C57BL/6 and C57BL/6 Pigr $^{-1-}$ mice were vaccinated with BPZE1 or left unvaccinated (naïve) as indicated. Four weeks later, mice were sacrificed and nasal washes were collected. Total IgG (a) and IgA (b) titers were measured against BPSM lysate. $N=5$. The nasal washes were transferred intranasally into naïve C57BL/6 mice, followed by challenge with $5 \times 10^{4} \mathrm{CFU}$ virulent $B$. pertussis BPSM. CFU levels were measured in the nose at the indicated time points (c). $N=3$ for the $3 \mathrm{~h}$ time point and 5 for the day 7 time point. Bars represent mean values and graph represents data combined from three independent experiments ${ }^{* * *} p<0.001$ by two-way ANOVA and Bonferroni post hoc test

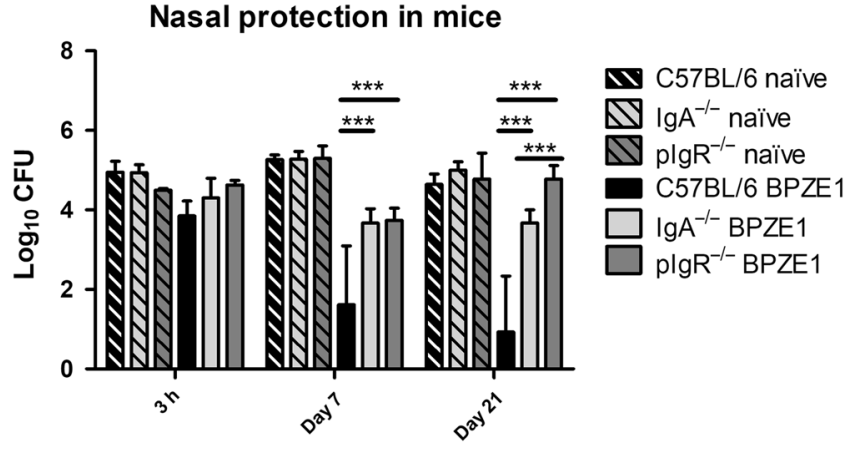

Fig. 5 BPZE1-induced protection against nasal colonization by $B$. pertussis in C57BL/6 mice and IgA $A^{-1-}$ and Pigr $^{-1-}$ strains. Groups of C57BL/6, C57BL/6 $\lg A^{-1-}$, and C57BL/6 $\mathrm{Pigr}^{-1-}$ mice were vaccinated with BPZE1 or left unvaccinated (naïve) as indicated, and 8 weeks later, mice were challenged with B. pertussis BPBCTA1 and CFU levels were measured in the nose at the indicated time points. $N=3$ for the $3 \mathrm{~h}$ time point and $N=5$ for the other time points. Bars represent mean values and graph represents data from a representative experiment. ${ }^{* *} p<0.001$ by two-way ANOVA and Bonferroni post hoc test the mucosal immune responses influence the outcome of the infection. However, surprisingly, most studies on adaptive immunity to pertussis have initially focused first on serum antibody responses, and then on circulating $T$ cells in humans and splenocytes in mice. ${ }^{25}$ Mucosal antibody and $T$ cell responses have so far attracted comparably little attention, although SIgA have been detected in mucosal secretions of $B$. pertussis-infected humans, ${ }^{26}$ and previous studies have suggested that they may contribute to protection. ${ }^{27}$ However, in other studies B. pertussis antigens formulated with mucosal adjuvants and delivered nasally to induce SIgA in mice did not confer better protection than when delivered parenterally. ${ }^{25}$ Furthermore, using $\lg A^{-1-}$ mice it has been suggested that $\lg A$ are not critical for protection of mice against nasal colonization by $B$. pertussis, although they appeared to be involved in reducing nasal infection, but not lung infection, by closely related Bordetella bronchiseptica. ${ }^{28}$ However, more recently, Raeven et al. ${ }^{29}$ have shown that pulmonary immunization with pertussis outer membrane vesicles induced superior protection than subcutaneous immunization and this was related to mucosal $\lg A$ and Th17 cell induction. The same authors have shown that mucosal $\lg A$ and Th1/Th17 cells are also induced by experimental infection of mice with virulent B. pertussis, ${ }^{30}$ and Th1/Th17 cells have been demonstrated to be crucial for protection induced by infection. ${ }^{31}$ Furthermore, Wilk et al. ${ }^{23}$ and 


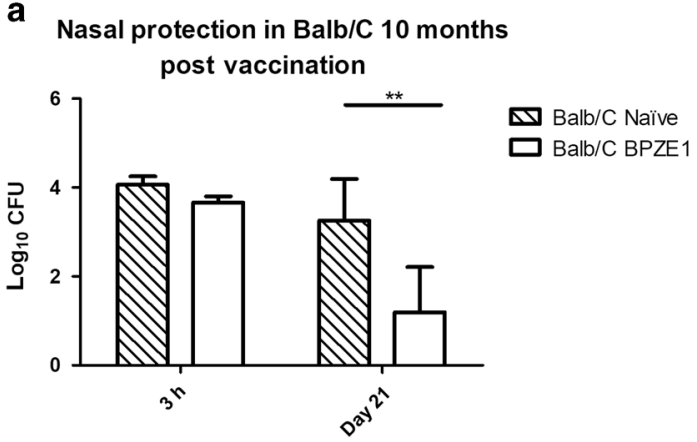

C Nasal protection in C57BL/6 10 months post vaccination

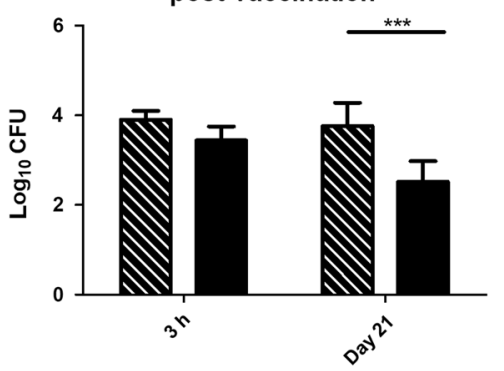

b

Challenge of Balb/C after nasal wash transfer: Nose

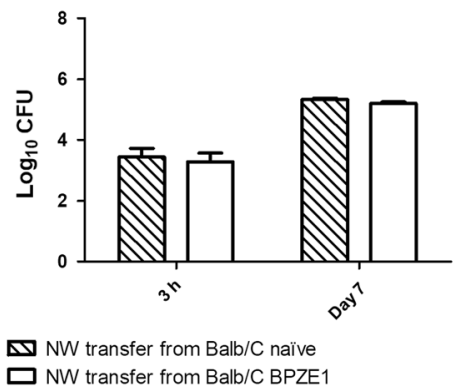

d

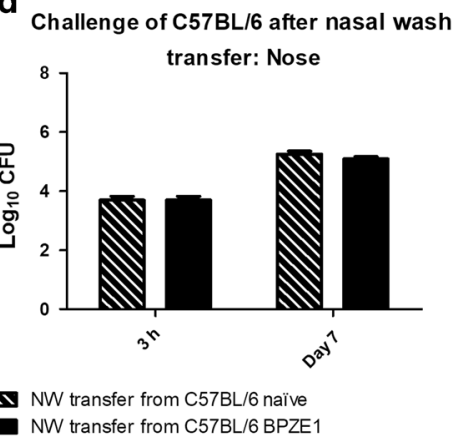

Fig. 6 BPZE1-mediated long-term protection against nasal colonization by B. pertussis in Balb/c and C57BL/6 mice. Groups of Balb/c and C57BL/6 mice were vaccinated with BPZE1 or left unvaccinated (naïve) as indicated. Ten months later, mice were challenged with $B$. pertussis BPBCTA1 and nasal CFU levels were measured at the indicated time points in Balb/c (a) and C57BL6 (c) mice. Ten months after vaccination, mice were sacrificed and nasal washes were collected and transferred intranasally into naïve Balb/c (b) or C57BL/6 (d) followed by challenge with $5 \times 10^{4} \mathrm{CFU}$ virulent $B$. pertussis BPSM. CFU levels were measured in the nose at the indicated time points. $N=3 \mathrm{for}$ the $3 \mathrm{~h}$ time point and $N=5$ for the other time points. Bars represent mean values and graph represents data from a representative experiment. ${ }^{* * *} p<0.001$ and ${ }^{* *} p$ $<0.01$ by two-way ANOVA and Bonferroni post hoc test

Misiak et al. ${ }^{32}$ have reported that infection with virulent B. pertussis induces tissue-resident IL-17-producing CD4 and $\gamma \delta \mathrm{T}$ cells in the lungs of mice. However, all these studies examined pulmonary or systemic T cell or IgA responses, and none of them addressed the responses in the nasal cavity.

Here, we found that aPV given subcutaneously protected mice very well against $B$. pertussis colonization of the lungs, although it did not induce any appreciable levels of serum IgA. In contrast, aPV provided no protection against $B$. pertussis colonization in the nasal cavity of the mice. This is reminiscent with what has been reported in the baboon model. ${ }^{12}$ It is also consistent with epidemiological studies in humans indicating that the currently used pertussis vaccines do not prevent nasopharyngeal B. pertussis infection and transmission. ${ }^{33}$ Incidentally, this may be the main reason for the failure of cocoon vaccination strategies to protect newborns against pertussis, even in settings in which these strategies have been successfully implemented. ${ }^{34}$

In contrast to aPV, a single dose of BPZE1 delivered nasally induced serum and local anti- $B$. pertussis IgA responses in addition to serum IgG and provided strong protection against $B$. pertussis colonization of both the lungs and the nose. This is in contrast to a previous study showing that prior infection with $B$. pertussis did not prevent subsequent nasal colonization by the same organism. ${ }^{28}$ The reasons for these conflicting findings are not known, but may potentially be due to the fact that we used the genetically attenuated BPZE1 strain, whereas in the previous study, virulent $B$. pertussis was used for the initial infection. However, our findings are consistent with our previous study showing BPZE1-induced protection against nasal infection in baboons, $^{16}$ in contrast to aPV. ${ }^{12}$

In the mice, the nasal anti-B. pertussis $\lg A$ induced by BPZE1 were genuine $\operatorname{SIgA}$, as they were not induced in $\mathrm{Pigr}^{-1-}$ mice, lacking the secretory component and therefore unable to actively secrete $\lg A$ into the mucosal lumen. Consistent with a previous study, ${ }^{35}$ BPZE1-induced or aPV-induced serum antibodies and splenocytes were able to transfer a significant level of protection against pulmonary infection by $B$. pertussis to naïve mice. We have previously shown that BPZE1-mediated protection by passive transfer of splenocytes is essentially due to $\mathrm{CD}^{+}{ }^{+} \mathrm{T}$ cells. ${ }^{35}$ However, here we found that none of them transferred any detectable level of protection against nasal colonization. Significant protection against nasal colonization could only be transferred by NWs from BPZE1-vaccinated mice. Again, this depended on SIgA, as NWs from BPZE1-vaccinated Pigr $^{-1-}$ mice did not transfer protection. Consistently, BPZE1 failed to protect against nasal $B$. pertussis infection in $\mathrm{Pigr}^{-1-}$ and in $\mathrm{IgA} \mathrm{A}^{-1-}$ mice, indicating that both the $\lg A$ isotype and their active transport into the airway lumen are required for protection against nasal carriage.

Mathematical modeling studies have concluded that asymptomatic carriage and transmission of $B$. pertussis may be the most parsimonious reason for the current resurgence of pertussis in high vaccine coverage countries. ${ }^{11}$ Our data suggest that in order to prevent asymptomatic carriage and consequently transmission of B. pertussis, the induction of potent SIgA responses in the nasopharynx may be required, and that this can be achieved by nasally delivered live attenuated pertussis vaccines, such as BPZE1. However, compared to $\lg$, IgA have a relatively short half-life, ${ }^{21}$ and an additional major problem of the current pertussis vaccines is the short duration of immunity elicited. ${ }^{36}$ We have previously shown that a single nasal dose of BPZE1 induces very long-lived protection against lung colonization by B. pertussis. ${ }^{37}$ Here we show that it also induced long-term protection against nasal infection, as 10 months after a single nasal dose of BPZE1 the mice 


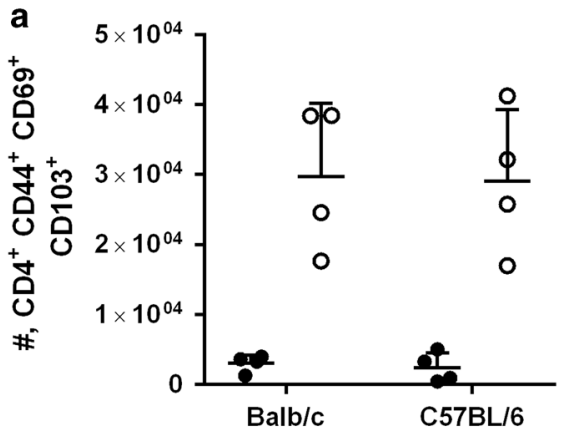

b
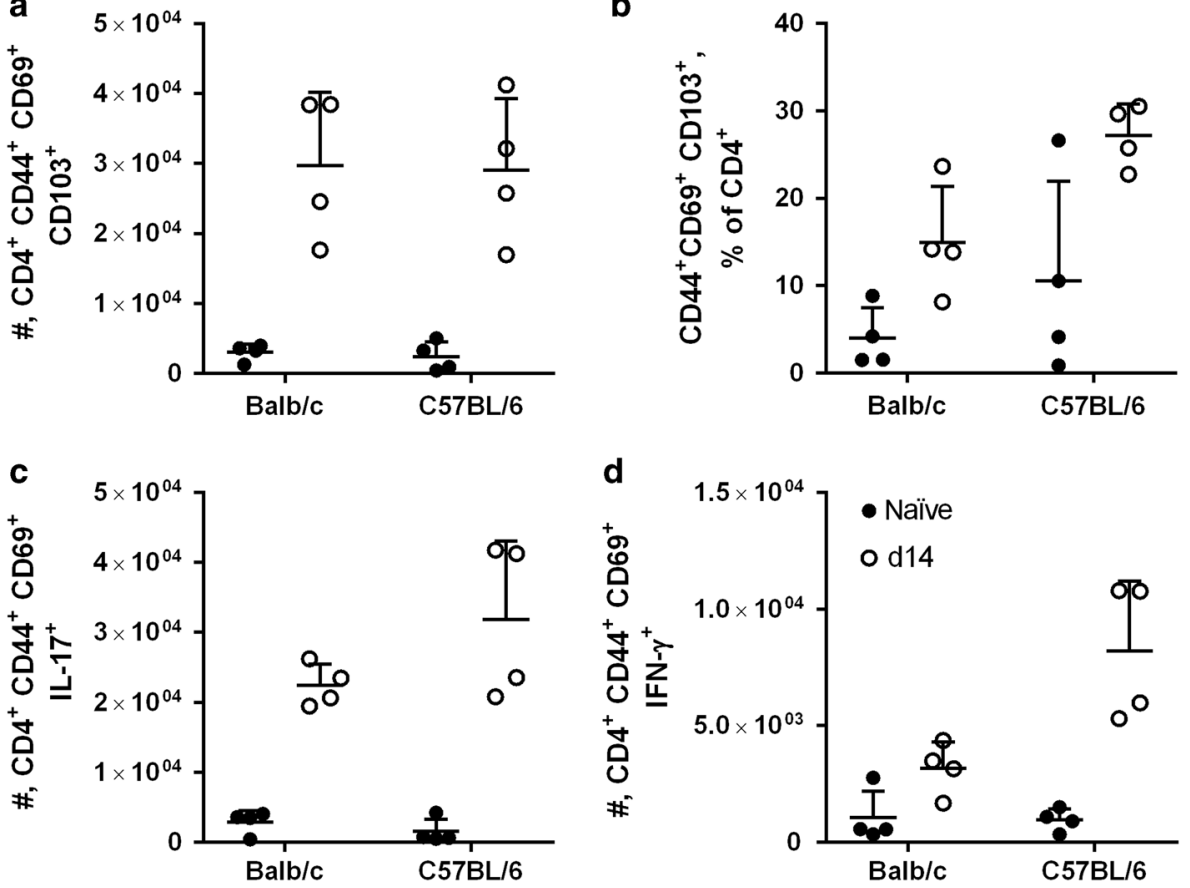

Fig. 7 Tissue-resident memory T (Trm) cells in nasal tissue post infection with BPZE1. C57BL/6 and Balb/c mice were infected intranasally with $10^{6}$ CFU BPZE1. Prior to (naïve) and 14 days post infection (d14), mice were i.v. injected with anti-CD45-PE antibody 10 min before euthanasia. Nasal tissue was taken and the T cell response was analyzed by flow cytometry. Only tissue-resident cells (CD45-PE negative) were included in the analysis. a Absolute counts of $\mathrm{CD}^{+} \mathrm{Trm}\left(\mathrm{CD} 45^{-}, \mathrm{CD} 44^{+}, \mathrm{CD} 69^{+}, \mathrm{CD} 103^{+}, \mathrm{CD} 4^{+}\right), \mathbf{b}$ percentages of Trm $\left(\mathrm{CD} 45-, \mathrm{CD} 44^{+}, \mathrm{CD} 69^{+}, \mathrm{CD} 103^{+}\right)$ of total $\mathrm{CD}^{+}{ }^{+}$cells, $\mathbf{c}$ absolute counts of IL-17-producing $\mathrm{CD}^{+}{ }^{+} \mathrm{Trm}, \mathbf{d}$ absolute counts of IFN- $\gamma$-producing $\mathrm{CD}^{+}{ }^{+} \mathrm{Trm}$. The lines indicate the means, the error bars show the SD

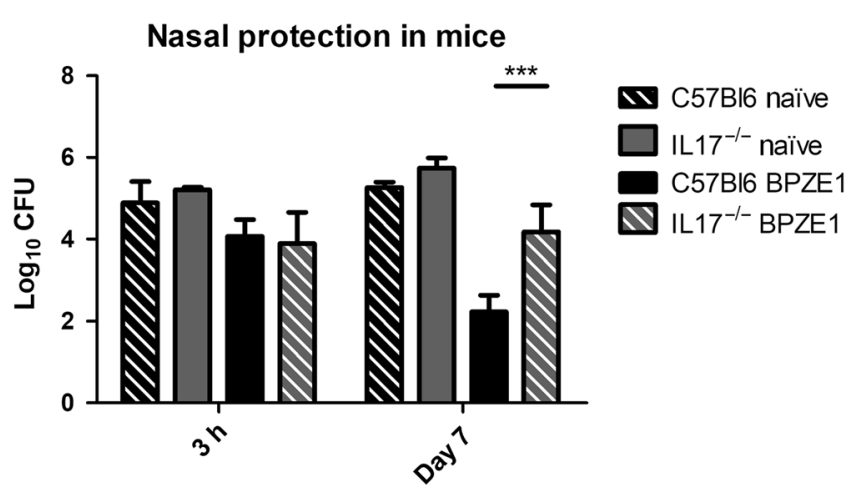

Fig. 8 BPZE1-mediated protection against nasal colonization by $B$. pertussis in C57BL/6 and $1117^{-1-}$ mice. Groups of C57BL/6 and C57BL/ $61117^{-1-}$ mice were vaccinated with BPZE1 or left unvaccinated (naïve) as indicated. Eight weeks later, mice were challenged with $B$. pertussis BPBCTA1 and CFU levels were measured in the nose at the indicated time points. $N=3$ for the $3 \mathrm{~h}$ time point and $N=5$ for the day 7 time point. Bars represent mean values and graph represents data from a representative experiment. ${ }^{* *} p<0.001$ by two-way ANOVA and Bonferroni post hoc test

still showed significant protection against nasal carriage. In contrast, NW harvested 10 months after vaccination failed to transfer any significant level of protection against nasal colonization, and B. pertussis-specific IgA levels in the NW had fallen to background levels 10 months after BPZE1 vaccination.

These observations indicate that in spite of the relatively fast $\lg A$ decay in the naso-pharynx, mucosal immune memory persists for a long time, potentially due to long-lived local IgA plasma cells. ${ }^{22}$ In order for these cells to produce the $B$. pertussis-specific IgA upon challenge infection, they are likely to require help from memory T cells. IL-17-producing Th17 cells have been implicated in both the IgA switch of plasma cells ${ }^{38}$ and the induction of $\mathrm{plgR}^{39}$ thereby optimizing SlgA production. We found that BPZE1 induces IL-17-producing $\mathrm{CD} 4{ }^{+} \mathrm{CD} 44^{+} \mathrm{CD} 69^{+}$Trm cells in the nose and that $1117^{-1-}$ mice were much less protected against nasal infection by $B$. pertussis upon BPZE1 vaccination. Furthermore, their level of specific IgA in the NWs was much reduced, compared to the NWs of BPZE1-vaccinated wild-type mice. These observations suggest thus that BPZE1-induced protective SIgA is dependent on IL-17-producing Trm cells. As they are long lived, it is therefore likely that these cells participate in sustaining BPZE1induced immune memory against nasal infection. In that regard, it is interesting to note that the long-term protection offered by BPZE1 vaccination against nasal infection was stronger in Balb/c mice than in C57BL/6 mice (see Fig. 6a, c), which is in line with the recent observation that spleenocytes from Balb/c mice produce more IL-17 upon stimulation with $B$. pertussis antigens than splenocytes from C57BL/6 mice. ${ }^{40}$

Long-lived IL-17-producing Th17 memory T cells have also been detected in the respiratory mucosa of $B$. pertussis-infected baboons, ${ }^{41}$ suggesting that the observations made in the mouse model can also be extended to primates. The $B$. pertussis-specific IL-17-producing memory $T$ cells persisted for at least 2 years after infection of the baboons. We have recently found that BPZE1 can also induce $\mathrm{IL}-17$ responses in baboons and that high levels of antigen-specific Th17 cells were associated with high $\lg \mathrm{A}$ responses against the surface antigen pertactin and strong sterilizing immunity in the baboon naso-pharynx $(\mathrm{CL}$, unpublished). It is not yet known whether BPZE1 also induces local Th17 lymphocyte responses in the naso-pharynx of humans. However, ex vivo studies have shown that BPZE1 can stimulate human monocyte-derived dendritic cells towards a Th17-polarizing phenotype, in a T cell-contact-dependent manner, ${ }^{42}$ which was shown to influence antiviral immune responses. ${ }^{43}$

In conclusion, we have found that, in contrast to aPV, BPZE1, in addition to protection against lung colonization, induces strong 
1760

protective immunity against nasal colonization by $B$. pertussis. BPZE1-mediated protection was dependent on the induction of specific SlgA secreted into the nasal cavity and on IL-17, most likely produced by Trm cells that appear to play a key role in sustaining mucosal adaptive immune memory elicited by nasal vaccination with BPZE1. Since the two major limitations of the current aPV are their inability to prevent $B$. pertussis infection and transmission and their short-lived immunity elicited, a vaccine, such as BPZE1, that provides both potent SIgA-based mucosal immunity in the naso-pharynx and that induces strong and longlasting local Th17 cell-based memory has the potential to be a major tool for the ultimate control of whooping cough.

\section{METHODS}

Bacterial strains and culture conditions

Bordetella pertussis BPZE $1,{ }^{13}$ BPSM, $^{44}$ and BPBCTA1, a BPSM derivative containing pFUS2 integrated in the $b c t A 1$ gene, ${ }^{45}$ used in this study, were cultured at $37^{\circ} \mathrm{C}$ on Bordet-Gengou (BG) agar, supplemented with $1 \%$ glycerol and 10\% defibrinated sheep blood, as described. ${ }^{13}$ After growth, the bacteria were harvested by scraping the plates and resuspending them in phosphatebuffered saline (PBS) at the desired density. When required, streptomycin was used at $100 \mu \mathrm{g} / \mathrm{ml}$ and gentamycin at $10 \mu \mathrm{g} / \mathrm{ml}$.

\section{Protection experiments}

Balb/c and C57BL/6 mice were purchased from Charles Rivers, $1 g A^{-/-46,47}$ and Pigr $^{-1-}$ mice ${ }^{48}$ came from University of Zaragoza, and the SCID and $1117^{-1-}$ mice were provided by Institute Pasteur of Lille. To determine the BPZE1-protective efficacy, eight 6-weekold wild-type mice were intranasally vaccinated with $10^{6}$ viable BPZE1 or vaccinated subcutaneously with $1 / 5$ of the human dose of aPV (Infanrix ${ }^{\circledast}$, GlaxoSmithKline) and boosted 4 weeks later with the same dose, as described previously. ${ }^{13}$ Unvaccinated mice served as control. Eight weeks after the first vaccination, the mice were challenged intranasally with $10^{6} \mathrm{CFU}$ of virulent BPSM or BPBCTA1. Three hours post challenge (day 0), 3 mice per group were sacrificed, and their lungs were harvested and homogenized for CFU measurement by plating 10-fold serial dilutions onto BG agar plates containing $10 \mu \mathrm{g} / \mathrm{ml}$ gentamycin, as previously described. ${ }^{13}$ In parallel, the noses of the mice were harvested as follows. Euthanized mice are put on their backs. After pulling off the nostril skin with tweezers, an incision was made on each side of the head between the nostrils and the cheeks and following the side of the head up to the neck to remove the skin from the top of the head. Then, an incision was made on each side of the head to cut and remove the lower jaw. Finally, incisions at the base of the palate and on each side of the head were made to remove the part of the head containing the nasal cavities. Residual skin, eyeball, and cheek muscle were carefully removed from the recovered tissues. The remaining material was then homogenized and plated out in serial dilutions as described above for the lungs. ${ }^{13}$ Five mice per group were then treated similarly at the indicated time points.

For long-term protection experiments, eight 6-week-old mice were vaccinated with $10^{6}$ CFU BPZE1 or left unvaccinated to serve as control. Ten months later, mice were challenged intranasally with $10^{6}$ CFU of virulent BPBCTA1 and bacterial load load was measured as described above at $3 \mathrm{~h}$ and 21 days post infection.

\section{Passive transfer experiments}

Groups of eight 6-week-old Balb/c mice were vaccinated with BPZE1 or aPV as described above. Eight weeks after the first vaccination, the mice were euthanized, and spleen and blood were collected. Splenocytes and sera were purified as described elsewhere before. ${ }^{37}$ One hundred microliters of serum or $5 \times 10^{7}$ total spleen cells were transferred intraperitoneally into SCID mice, which were challenged intranasally with $10^{6} \mathrm{CFU}$ of virulent
BPBCTA1 $24 \mathrm{~h}$ later. CFUs were measured at the indicated time points in the lungs and noses as described above.

For passive transfer of mucosal antibodies, eight 6-week-old mice were vaccinated intranasally by BPZE1 or left unvaccinated. Four weeks or 10 months later, the mice were euthanized and the NW were harvested by flushing PBS with a cocktail of antiproteases complete mini (Roche) through the nose and concentrating the washes by using $10-\mathrm{kDa}$ cut-off Amicon ${ }^{\circledR}$ Ultra columns (Millipore) following the manufacturer's instructions. Five microliters of the concentrated NW was then transferred intranasally into the noses of 6 -week-old naïve $\mathrm{C} 57 \mathrm{BL} / 6$ mice, followed by intranasal challenge with $5 \times 10^{4} \mathrm{CFU}$ virulent BPSM and CFU counting in the nose $3 \mathrm{~h}$ and 7 days post challenge as described above. We used an approximately 10 -fold lower challenge dose compared to the other challenge experiments, as due to the low amount of antibodies in the NW, a high challenge dose would have been too overwhelming to see a potential protective effect of NW antibodies.

\section{Antibody titer determination}

To determine antibody titers in the sera and NWs, standard enzyme-linked immunosorbent assay (ELISA) protocols were used. Briefly, 96-well plates (Nunc) were coated with $1 \mu \mathrm{g}$ per well of total $B$. pertussis BPSM lysate or $1 \mu \mathrm{g}$ per well PTX, FHA, or pertactin and then blocked with $2 \%$ bovine serum albumin in PBS $/ 0.5 \%$ Tween. BPSM lysates were prepared from a 200-ml bacterial culture grown in Stainer Scholte medium until stationary phase. The bacteria were then harvested by centrifugation, resuspended in $10 \mathrm{ml}$ PBS with a cocktail of anti-proteases complete mini (Roche), and lysed by serial passages through the French press. The protein concentration was quantified using BCA test (Thermo Fisher) following the manufacturer's instructions. The coated plates were then incubated for $2 \mathrm{~h}$ at $37^{\circ} \mathrm{C}$ with serum or NW samples in twofold serial dilutions in PBS/0.5\% Tween. After three washes with PBS/0.5\% Tween, horse radish peroxidase (HRP)-goat anti-mouse lgG (1:5000) (Sigma), HRP-goat anti-mouse IgA (1:500) (Sigma), HRP-goat anti-mouse IgG1 (1:1000) (Southern Biotech), or HRP-goat anti-mouse IgG2a $(1: 20,000)$ (Southern Biotech) was added and the plates were incubated at room temperature for $1 \mathrm{~h}$. After washing five times with $\mathrm{PBS} / 0.5 \%$ Tween, the reaction was developed by using TMB (Interchim) and $1 \mathrm{M} \mathrm{H}_{3} \mathrm{PO}_{4}$ and the plates were read using BioTek ELx8000.

Determination of IL-17 secretion after antigen stimulation Lung cells were prepared from lung homogenates by Percoll gradient centrifugation and resuspended in RPMI medium containing $10 \%$ fetal calf serum. Total splenocytes were isolated as described. ${ }^{13}$ The cells were then stimulated for $72 \mathrm{~h}$ with $1 \mu \mathrm{g}$ of PTX, FHA, or pertactin at $37{ }^{\circ} \mathrm{C}$ and $5 \% \mathrm{CO}_{2}$. The supernatants were then harvested and IL-17A was measured by ELISA using the mouse IL-17A ELISA Kit (Mabtech, Sweden).

Isolation and flow cytometry analysis of cells from nasal tissue Ten minutes before euthanasia, mice were intravenously injected with $7.5 \mu \mathrm{g}$ anti-CD45-PE antibody (ebiosciences) to allow for the distinction of circulating $T$ cells (CD45-PE positive) and resident T cells (CD45-PE negative). Nasal tissue was scraped from the nasal cavity, digested with collagenase-D ( $1 \mathrm{mg} / \mathrm{ml}$; Roche) and DNase I (10 mg/ml; Sigma-Aldrich) for $1 \mathrm{~h}$ at $37^{\circ} \mathrm{C}$ with agitation, and then passed through a cell strainer. Cells were stimulated with phorbol myristate acetate (PMA) $(50 \mathrm{ng} / \mathrm{ml})$ and ionomycin $(500 \mathrm{ng} / \mathrm{ml})$ in the presence of brefeldin A $(5 \mathrm{mg} / \mathrm{ml})$ for $4 \mathrm{~h}$ at $37^{\circ} \mathrm{C}$. After incubation with LIVE/DEAD Aqua (Invitrogen), cells were surface stained with the following fluorochrome-conjugated antibodies: CD69-FITC, CD8-AF700, CD3-APC-ef780 (eBiosciences), CD44BV605, CD4-BV785 (BioLegend), and CD103-PE-CF594 (BD Biosciences). For the detection of intracellular cytokines, cells were fixed and permeabilized using a FoxP3 Transcription Factor 
staining buffer set (ebiosciences), and stained with the following antibodies: IL-17-V450 (BD Biosciences) and IFN- $\gamma-\mathrm{PE}-\mathrm{Cy} 7$ (ebiosciences). Fluorescence minus one samples were used as controls. Fluorescence-activated cell sorting samples were acquired on a LSR Fortessa using the BD Diva Software (BD Biosciences) and analyzed using the Flowjo Software (v10, TreeStar).

Statistical analyses

Statistical analyses were performed by one-way analysis of variance (ANOVA) or two-way ANOVA and Bonferroni post hoc test using the GraphPad Prism software. $P$ values $<0.05$ were considered significant.

Ethical statement

All the animal experiments were carried out in accordance with the guidelines of the French Ministry of Research regarding animal experiments, and the protocols were approved by the Ethical Committees of the Region Nord Pas de Calais and the Ministry of Research (agreement number APAFIS\#9107士201603311654342 V3).

\section{ACKNOWLEDGEMENTS}

Part of this work has been financially supported by ILiAD Biotechnologies. K.H.G.M. is supported by Science Foundation Ireland (grant \# 16/IA/4468).

\section{AUTHOR CONTRIBUTIONS}

L.S., N.A., F.T., C.M. and C.L. designed the experiments; L.S., A.-S.D., A.T., L.C. and S.U. performed the experiments; L.B. and K.H.G.M. performed the Trm experiments, L.S., A.-S.D., N.A., C.M. and C.L. analyzed the data; L.S. and C.L. wrote the manuscript

\section{ADDITIONAL INFORMATION}

The online version of this article (https://doi.org/10.1038/s41385-018-0073-9) contains supplementary material, which is available to authorized users.

Competing interest: A.-S.D., C.L. and L.S. are inventors of patents on the BPZE1 vaccine. The other authors declare no competing interests.

\section{REFERENCES}

1. Mattoo, S. \& Cherry, J. D. Molecular pathogenesis, epidemiology, and clinical manifestations of respiratory infections due to Bordetella pertussis and other Bordetella subspecies. Clin. Microbiol. Rev. 18, 326-382 (2005).

2. Hewlett, E. L. \& Edwards, K. M. Clinical practice. Pertussis-not just for kids. N. Engl. J. Med. 352, 1215-1222 (2005).

3. World Health Organization. Global routine vaccination coverage, 2010. Wkly Epidemiol. Rec. 2011; 86: 509-513.

4. Cherry, J. D. Epidemic pertussis in 2012-the resurgence of a vaccine-preventable disease. N. Engl. J. Med. 367, 785-787 (2012).

5. Burns, D. L., Meade, B. D. \& Messionnier, N. E. Pertussis resurgence: perspectives from the Working Group Meeting on pertussis on the causes, possible paths forward, and gaps in our knowledge. J. Infect. Dis. 209(Suppl. 1), S32-S35 (2014).

6. Zhang, Q. et al. Prevalence of asymptomatic Bordetella pertussis and Bordetella parapertussis infections among school children in China as determined by pooled real-time PCR: a cross-sectional study. Scand. J. Infect. Dis. 46, 280-287 (2014).

7. de Greeff, S. C. et al. Seroprevalence of pertussis in The Netherlands: evidence for increased circulation of Bordetella pertussis. PLOS ONE 5, e14183 (2010).

8. Hodder, S. L. et al. Antibody responses to Bordetella pertussis antigens and clinical correlations in elderly community residents. Clin. Infect. Dis. 31, 7-14 (2000).

9. Moriuchi, T., Otsuka, N., Hiramatsu, Y., Shibayama, K. \& Kamachi, K. A high seroprevalence of antibodies to pertussis toxin among Japanese adults: qualitative and quantitative analyses. PLOS ONE 12, e0181181 (2017).

10. Scott, S. et al. Seroprevalence of pertussis in the Gambia: evidence for continued circulation of bordetella pertussis despite high vaccination rates. Pediatr. Infect. Dis. J. 34, 333-338 (2015).

11. Althouse, B. M. \& Scarpino, S. V. Asymptomatic transmission and the resurgence of Bordetella pertussis. BMC Med. 13, 146 (2015).

12. Warfel, J. M., Zimmerman, L. I. \& Merkel, T. J. Acellular pertussis vaccines protect against disease but fail to prevent infection and transmission in a nonhuman primate model. Proc. Natl. Acad. Sci. USA 111, 787-792 (2014).
13. Mielcarek, N. et al. Live attenuated $B$. pertussis as a single-dose nasal vaccine against whooping cough. PLoS Pathog. 2, e65 (2006).

14. Locht, C. \& Mielcarek, N. Live attenuated vaccines against pertussis. Expert. Rev. Vaccin. 13, 1147-1158 (2014)

15. Thorstensson, R. et al. A phase I clinical study of a live attenuated Bordetella pertussis vaccine-BPZE1; a single centre, double-blind, placebo-controlled, doseescalating study of BPZE1 given intranasally to healthy adult male volunteers. PLOS ONE 9, e83449 (2014).

16. Locht $C$. et al. Live attenuated pertussis vaccine BPZE1 protects baboons against B. pertussis disease and infection. J. Infect. Dis. 216, 117-124 (2017).

17. Guiso, N., Capiau, C., Carletti, G., Poolman, J. \& Hauser, P. Intranasal murine model of Bordetella pertussis infection. I. Prediction of protection in human infants by acellular vaccines. Vaccine 17, 2366-2376 (1999).

18. Mills, K. H., Ryan, M., Ryan, E. \& Mahon, B. P. A murine model in which protection correlates with pertussis vaccine efficacy in children reveals complementary roles for humoral and cell-mediated immunity in protection against Bordetella pertussis. Infect. Immun. 66, 594-602 (1998).

19. Mielcarek, N., Debrie, A.-S., Mahieux, S. \& Locht, C. Dose response of attenuated Bordetella pertussis BPZE1-induced protection in mice. Clin. Vaccin. Immunol. 17, 317-324 (2010).

20. Higgs, R., Higgins, S. C., Ross, P. J. \& Mills, K. H. Immunity to the respiratory pathogen Bordetella pertussis. Mucosal Immunol. 5, 485-500 (2012).

21. Delacroix, D. L. et al. Changes in size, subclass, and metabolic properties of serum immunoglobulin $A$ in liver diseases and in other diseases with high serum immunoglobulin A. J. Clin. Invest. 71, 358-367 (1983).

22. Spencer, J. \& Sollid, L. M. The human intestinal B-cell response. Mucosal Immunol. 9, 1113-1124 (2016).

23. Wilk, M. M. et al. Lung CD4 tissue-resident memory T cells mediate adaptive immunity induced by previous infection of mice with Bordetella pertussis. J. Immunol. 199, 233-243 (2017).

24. Paddock, C. D. et al. Pathology and pathogenesis of fatal Bordetella pertussis infection in infants. Clin. Infect. Dis. 47, 328-338 (2008).

25. Mills, K. H. Immunity to Bordetella pertussis. Microbes Infect. 3, 655-677 (2001).

26. Zackrisson, G., Lagergard, T., Trollfors, B. \& Krantz, I. Immunoglobulin A antibodies to pertussis toxin and filamentous hemagglutinin in saliva from patients with pertussis. J. Clin. Microbiol. 28, 1502-1505 (1990).

27. Hellwig, S. M., van Spriel, A. B., Schellekens, J. F., Mooi, F. R. \& van de Winkel, J. G. Immunoglobulin A-mediated protection against Bordetella pertussis infection. Infect. Immun. 69, 4846-4850 (2001).

28. Wolfe, D. N., Kirimanjeswara, G. S., Goebel, E. M. \& Harvill, E. T. Comparative role of immunoglobulin A in protective immunity against the Bordetellae. Infect. Immun. 75, 4416-4422 (2007).

29. Raeven, R. H. et al. Molecular and cellular signatures underlying superior immunity against Bordetella pertusiss upon pulmonary vaccination. Mucosal Immunol. 11, 979-993 (2018).

30. Raeven, R. H. et al. Immunological signatures after Bordetella pertussis infection demonstrates importance of pulmonary innate immune cells. PLOS ONE 11, e0164027 (2016).

31. Ross, P. J. et al. Relative contribution of Th1 and Th17 cells in adaptive immunity to Bordetella pertussis: towards the rational design of an improved acellular pertussis vaccine. PLoS Pathog. 9, e1003264 (2013).

32. Misiak, A., Wilk, M. M., Raverdeau, M. \& Mills, K. H. IL-17-producing innate and pathogen-specific tissue resident memory gammadelta $T$ cells expand in the lungs of Bordetella pertussis-infected mice. J. Immunol. 198, 363-374 (2017).

33. Locht, C. Live pertussis vaccines: will they protect against carriage and spread of pertussis? Clin. Microbiol. Infect. 22(Suppl. 5), S96-S102 (2016).

34. Healy, C. M., Rench, M. A., Wootton, S. H. \& Castagnini, L. A. Evaluation of the impact of a pertussis cocooning program on infant pertussis infection. Pediatr. Infect. Dis. J. 34, 22-26 (2015).

35. Feunou, P. F., Bertout, J. \& Locht, C. T- and B-cell-mediated protection induced by novel, live attenuated pertussis vaccine in mice. Cross protection against parapertussis. PLOS ONE 5, e10178 (2010).

36. Witt, M. A., Katz, P. H. \& Witt, D. J. Unexpectedly limited durability of immunity following acellular pertussis vaccination in preadolescents in a North American outbreak. Clin. Infect. Dis. 54, 1730-1735 (2012).

37. Feunou, P. F., Kammoun, H., Debrie, A. S., Mielcarek, N. \& Locht, C. Long-term immunity against pertussis induced by a single nasal administration of live attenuated B. pertussis BPZE1. Vaccine 28, 7047-7053 (2010).

38. Christensen, D., Mortensen, R., Rosenkrands, I., Dietrich, J. \& Andersen, P. Vaccineinduced Th17 cells are established as resident memory cells in the lung and promote local IgA responses. Mucosal Immunol. 10, 260-270 (2017).

39. Jaffar, Z., Ferrini, M. E., Herritt, L. A. \& Roberts, K. Cutting edge: lung mucosal Th17mediated responses induce polymeric lg receptor expression by the airway epithelium and elevate secretory IgA levels. J. Immunol. 182, 4507-4511 (2009). 
IL-17-dependent SlgA-mediated protection against nasal Bordetella... $\mathrm{L}$ Solans et al.

1762

40. Mosley Y. C., Lu F. \& HogenEsch H. Differences in innate IFNgamma and IL-17 responses to Bordetella pertussis between $\mathrm{BALB} / \mathrm{C}$ and $\mathrm{C} 57 \mathrm{BL} / 6$ mice: role of gammadeltaT cells, NK cells, and dendritic cells. Immunol Res. 65, 1139-1149, (2017).

41. Warfel, J. M. \& Merkel, T. J. Bordetella pertussis infection induces a mucosal IL-17 response and long-lived Th17 and Th1 immune memory cells in nonhuman primates. Mucosal Immunol. 6, 787-796 (2013).

42. Fedele, G., Bianco, M., Debrie, A. S., Locht, C. \& Ausiello, C. M. Attenuated Bordetella pertussis vaccine candidate BPZE1 promotes human dendritic cell CCL21induced migration and drives a Th1/Th17 response. J. Immunol. 186, 5388-5396 (2011).

43. Schiavoni, I. et al. Live attenuated $B$. pertussis BPZE1 rescues the immune functions of respiratory syncytial virus infected human dendritic cells by promoting Th1/Th17 responses. PLOS ONE 9, e100166 (2014).
44. Menozzi, F. D. et al. Heparin-inhibitable lectin activity of the filamentous hemagglutinin adhesin of Bordetella pertussis. Infect. Immun. 62, 769-778 (1994).

45. Antoine, R. et al. The periplasmic binding protein of a tripartite tricarboxylate transporter is involved in signal transduction. J. Mol. Biol. 351, 799-809 (2005).

46. Harriman, G. R. et al. Targeted deletion of the IgA constant region in mice leads to IgA deficiency with alterations in expression of other Ig isotypes. J. Immunol. 162, 2521-2529 (1999)

47. Mbawuike, I. N. et al. Mucosal immunity to influenza without $\lg \mathrm{A}$ : an $\lg \mathrm{A}$ knockout mouse model. J. Immunol. 162, 2530-2537 (1999).

48. Johansen, F. E. et al. Absence of epithelial immunoglobulin A transport, with increased mucosal leakiness, in polymeric immunoglobulin receptor/secretory component-deficient mice. J. Exp. Med. 190, 915-922 (1999). 